\title{
Severe Acute Respiratory Infections during the Influenza A(H1N1)2009 pandemic in Belgium: first experience of hospital-based flu surveillance
}

by

\author{
Hammadi $S^{1}$, Gutiérrez $I^{1,2}$, Litzroth $A^{1}$, Mertens $K^{1}$, Wuillaume $F^{1}$
}

\begin{abstract}
Introduction

In September 2009, as part of the surveillance during the Influenza A(2009) pandemic, Belgium introduced a web-based surveillance system aimed at recording hospitalisations and deaths attributable to Influenza in real time.

Methods

We present the web-based application developed for the pandemic as well as a descriptive analysis of Severe Acute Respiratory Infection (SARI) cases reported through this system.

Results

From 1 September to 31 December 2009, 1723 SARI-related hospitalisations potentially due to influenza were reported in Belgium. The median age of the patients was 29 years (range: $<1$ year-99 years). Among SARI-hospitalised patients 68\% were aged less than 45 years, $10.6 \%$ were vaccinated with the seasonal influenza vaccine and $7.5 \%$ with the pandemic influenza vaccine. No deaths were recorded.
\end{abstract}

\section{Conclusions}

This first experience showed the feasibility of getting real-time information from hospitals during a public health crisis. However, the absence of death detected through the system highlighted the importance of better defining the severity of the hospital cases.

\section{Keywords}

SARI, hospitalisation, surveillance, influenza $A(H 1 N 1) 2009$, epidemiology

\section{Introduction}

From April 2009, the reports on the first cases of Influenza A(H1N1)2009 showed a moderate severity of the disease (1-2), most patients experiencing a self-limited illness, except in some

\footnotetext{
Scientific Institute of Public Health, Directorate Public Health and Surveillance, Brussels, Belgium

2 European Programme for Intervention Epidemiology Training (EPIET), European Centre for Disease Prevention and Control, Stockholm, Sweden

samia.hammadi@wiv-isp.be
} 
groups at higher risk of severe disease and deaths (3-4). The monitoring of the severity of the new variant influenza by Member States was highly recommended by the ECDC and the WHO (3-5). In Belgium, no system for reporting influenza-related hospitalised cases and influenza-specific deaths existed before the pandemic.

Hospital-based surveillance for severe acute respiratory infections (SARI) can help in characterising the risks of clinical disease, severity and death, and in identifying the risk groups and risk settings (6). Drawing a picture of severe cases asks for a complex and demanding hospital-based surveillance system. However, in the early phase of the pandemic, a significant increase in the workload of emergency wards was expected (1-4), limiting the possibility to add tasks for surveillance and reporting. The hospital representatives required a light set of variables to be recorded at the entry point of the hospital only. With this constraint, no followup of the patients within the hospital was possible and the clinical outcomes of the patient, such as deaths, could not be recorded.

The system aimed at recording all hospitalisations for SARI during the Influenza $A(H 1 N 1) 2009$ pandemic. This paper describes the surveillance system and the weekly incidence of SARI cases by age and geographical area from week 39 to week 53 in 2009 in Belgium.

\section{Methods}

In August, pilot hospitals tested a web-based application known as 'eH1N1'. Next, the Scientific Institute of Public Health (WIV-ISP) invited the 147 Belgian hospitals with emergency wards to participate, on a voluntary base, in the SARI surveillance.

The surveillance started on 1 September 2009. It consisted of real-time notifications of SARI admissions in the web-based application eH1N1. The date of admission, the vaccine status for seasonal and pandemic influenza, and the national number of the patient were collected. From the national number, the eH1N1 application deduced the year of birth and the postcode of residence of the patient. WIV-ISP was provided with a code unique per patient, which permitted the identification of double entries and multiple hospitalisations. Due to privacy protection constraints, the sex of the patient was not transmitted.

The eH1N1 application checked the vital status of the patients by linking the national number of SARI patients to the national register of the population. The death of a patient could be detected up to two months after his or her admission.

Each week, WIV-ISP sent a recall mail to the person in charge of the surveillance in the hospital. We asked the hospitals to explicitly report zero cases when they had no SARI hospitalisations, to make sure no cases were omitted or forgotten.

Results were presented in the weekly Belgian Influenza bulletin and published on the internet. 
We used the WHO definition for SARI: a person with sudden onset of fever $>38^{\circ} \mathrm{C}$ and cough or sore throat in the absence of any other diagnosis and shortness of breath or difficulty breathing and requiring hospital admission (6).

In order to introduce this surveillance system, a formal ethical approval was obtained from the Belgian Commission for the protection of privacy.

Data analysis

We carried out descriptive statistics for all study variables. All cases were included in the analysis. We categorised patients according to age groups. Statistical analysis was conducted using Stata v10 (StataCorp, College Station, TX, USA).

\section{Results}

During the period from 1 September 2009 to the end of December 2009, 1723 hospitalisations due to SARI were reported. Among all Belgian hospitals $(n=147), 85(60.3 \%)$ agreed to participate in this surveillance and an average of 40 (range 39-53) hospitals reported cases of SARI every week. This number varied from week to week. The number of notified SARI increased from week 40 to week 45 consistently with the epidemic curve of ILI (Figure 1) and the mean weekly number reported per hospital varied from 0.9 to 3.8 at the peak.

Figure 1. Number of SARI hospitalisations and ILI incidence by week, Belgium, September-December 2009

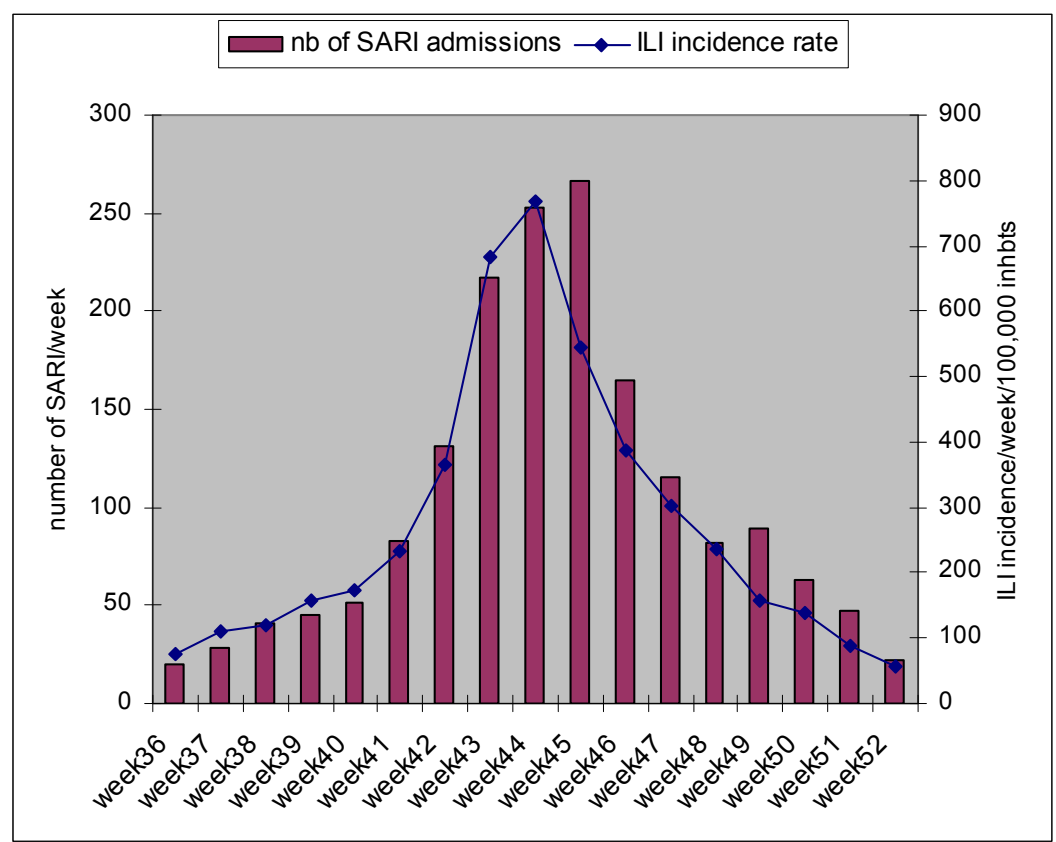

The median age of all cases was 29 years (range $<1$ year to 99 years). Sixty-eight percent of cases appeared to be people under 45 years of age. Most SARI cases were children, and the 0 to 5 year group represented $33.2 \%$ of all SARI. 
The overall rate of hospitalisation per million inhabitants was 169, ranging from 153 in those aged 85 years or older to 967 in infants younger than 5 years (Figures 2 and 3 ).

Figure 2. SARI incidence per million inhabitants, by age group, Belgium, September-December 2009

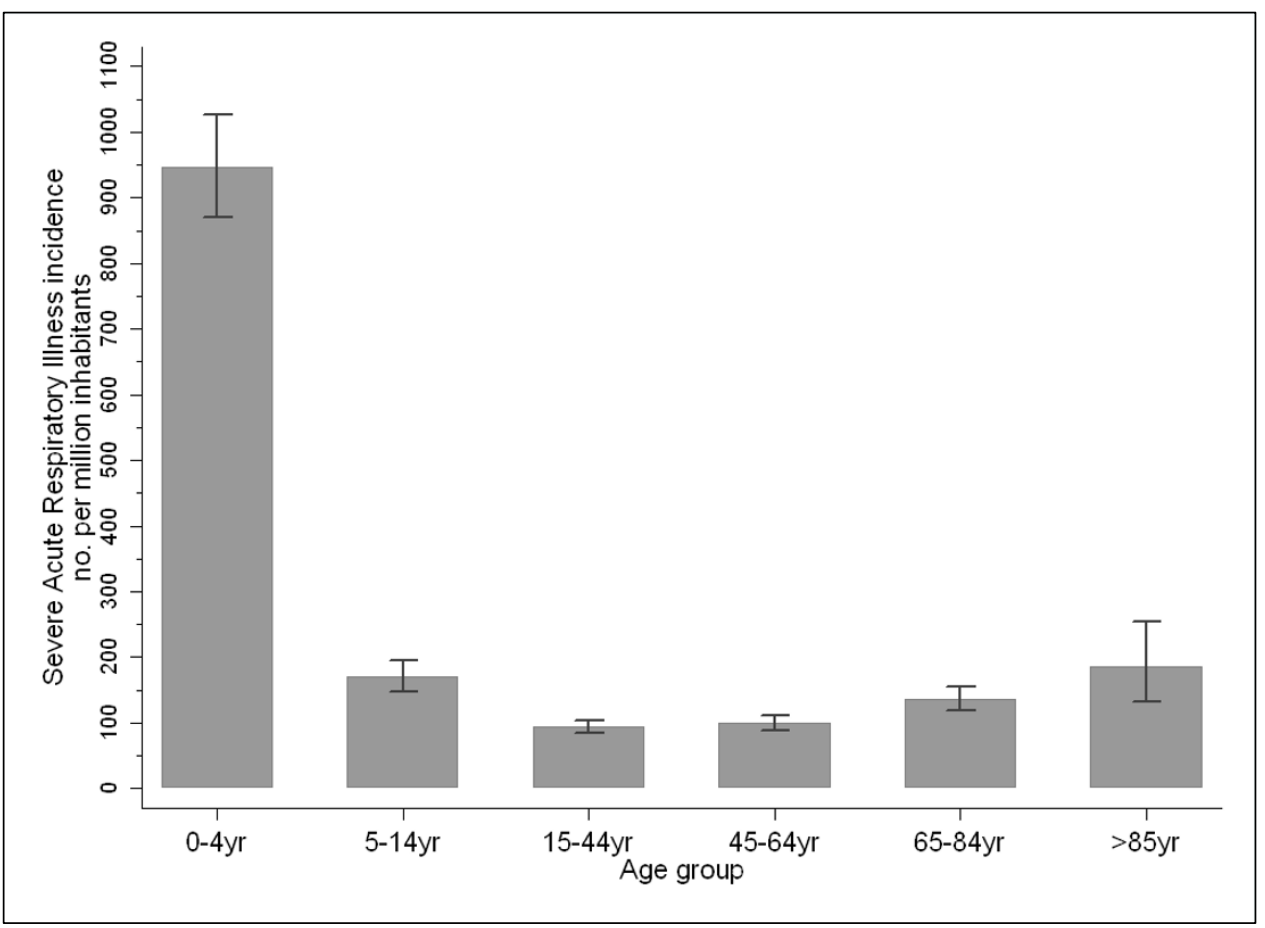

Figure 3. Weekly SARI hospitalisation rates per age group (September-December 2009)

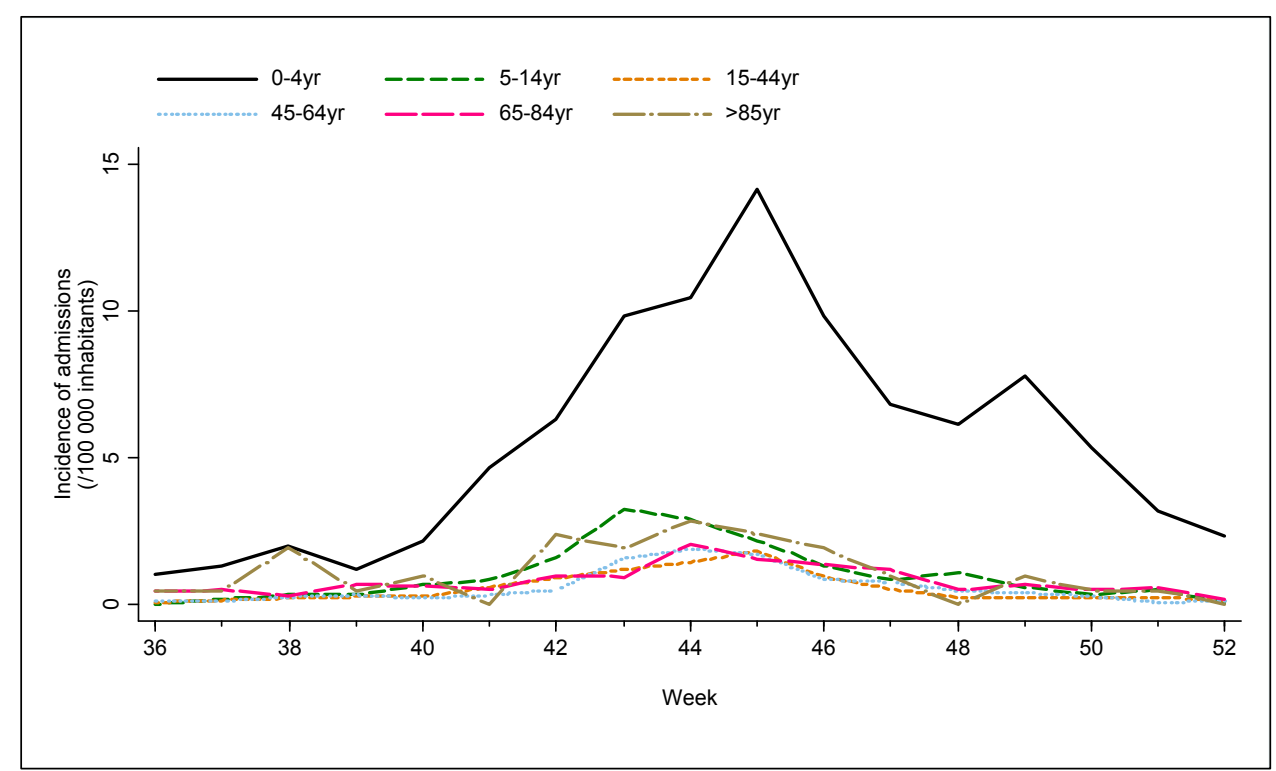

In Belgium, the vaccination against seasonal influenza started in week 40 (beginning of October) and the vaccination against pandemic influenza started in week 45 for the health staff and week 47 (mid-November) for the populations at higher risk. Among SARI cases hospital- 
ised from mid-October, $10.6 \%$ (67/630) were vaccinated with the seasonal trivalent influenza vaccine. Among SARI cases hospitalised from mid-November, $7.5 \%(13 / 173)$ had been vaccinated with the new pandemic influenza vaccine.

The system did not report any deaths among patients hospitalised during this period and registered in the surveillance database.

\section{Discussion}

This was the first attempt of the Belgian Influenza surveillance system to record SARI potentially attributed to influenza. From conception, the system wanted to limit additional work in emergency wards for surveillance purposes. The timeliness of reporting was a major benefit of the system, balanced by a lack of information to complete a description of hospitalised cases.

The participation in the surveillance was voluntary and we observed a strong heterogeneity in the reporting among regions. A higher proportion of Flemish hospitals reported cases than in other regions (Brussels, Wallonia) (Figure 4).

Figure 4. Reporting of Severe Acute Respiratory Illness in Belgium, fall 2009:

percentage of hospitals that reported data on SARI per province

for at least 1 week(s)

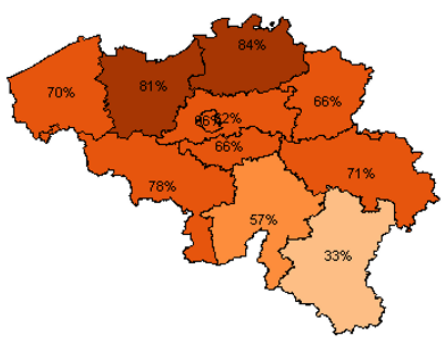

for at least 10 week(s)

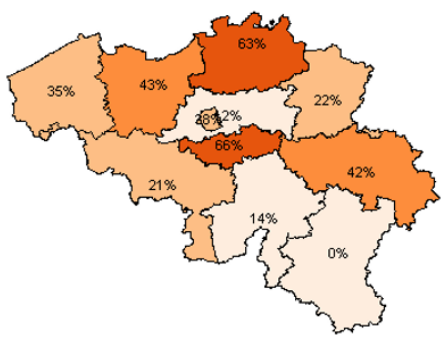

for at least 5 week(s)

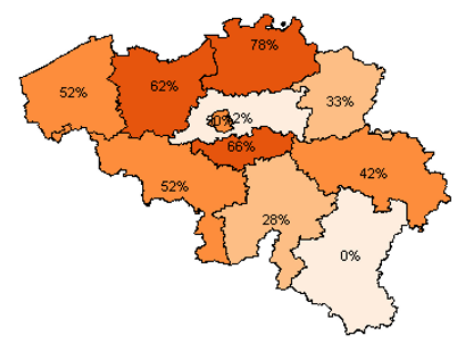

for at least 15 week(s)

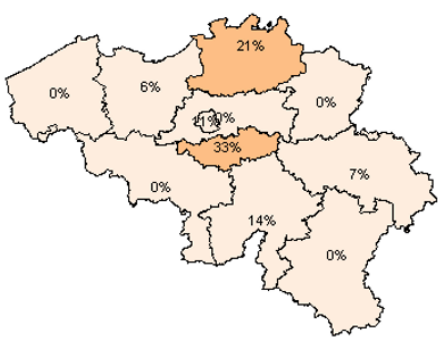

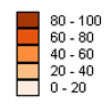

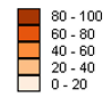


The median age of hospital cases was 29 years, lower than expected in seasonal influenza (7-9). Most hospital admissions were observed in patients younger than 45 year (7-10). In our series, the SARI hospitalisation rate per 100,000 was comparable among age groups, ranging from 10.4 to 19.1 , with the exception of infants who had the highest rate with 95.4 SARI hospital admissions per 100,000 inhabitants. Data collected by the surveillance do not permit to score the severity of hospitalised patients, especially among children. Some of the children were probably hospitalised due to precautionary measures rather than to severe complications (11-12).

The results were consistent with those found in other studies (3, 13-15). However, our case definition of SARI was not specific and did not require laboratory case confirmation, which could explain a higher hospitalisation rate in our series.

Changes in SARI hospitalisation rates may provide information on the burden of the epidemic in hospital services. One of the major shortcomings in our system was the lack of additional criteria to score the severity of the cases. Data were collected at the entry point of the hospital and no follow-up information was collected. The duration of the hospital stay, rates of complications and/or outcomes and admission to intensive care units were thus unknown. It was only possible to relate SARI patients to the death register. Strangely enough, none of our patients died in the two months following their hospital admission. This absence of mortality in our SARI population is highly questionable. One hypothesis is that severity was not a major criterion leading to hospitalisation in our series. Another possibility is that a more specific case definition is necessary. Yet, the trends of SARI were perfectly consistent with the trends of ILI recorded by the sentinel network of general practitioners (16).

\section{Conclusions}

This experience of SARI surveillance in Belgium showed the feasibility of getting real-time information from hospitals. This is important for managing health-related crises such as pandemic influenza. However, the current system did not provide sufficient information on the severity of SARI cases and this experience demonstrated that collecting data on hospitalisation only is insufficient to score the severity of an event. To define those severity criteria while respecting the workload and organisational constraints of emergency services will constitute the next challenge for the monitoring of public health events.

\section{References}

1. World Health Organization (WHO). What is phase 6 ? What is about severity?. Available from: http://www.who.int/csr/disease/swineflu/frequently asked questions/levels pandemic alert/en/index.html

2. World Health Organization (WHO). Assessing the severity of on influenza pandemic. Available from: http://www.who.int/csr/disease/swineflu/assess/disease swineflu assess 20090511/en/index.html

3. European Centre of Disease Prevention and Control. ECDC Risk assessment. 2009 influenza A (H1N1) pandemic. Version 7. 17 December 2009. Available from:

http://www.ecdc.europa.eu/en/healthtopics/Documents/0908 Influenza AH1N1 Risk Assessment.pdf 
4. Belgian working group on influenza $A(H 1 N 1) v$. Influenza $A(H 1 N 1) v$ virus infections in Belgium, May-June 2009. Euro Surveill. 2009;14(28):pii=19270. Available from: http://www.eurosurveillance.org/ViewArticle.aspx?Articleld $=19270$

5. World Health Organization (WHO). Weekly epidemiological record 24 July 2009, vol. 84, 30 (pp 301 308). Available from: http://www.who.int/wer/2009/wer8430/en/index.html

6. World Health Organization. Human infection with pandemic (H1N1)2009 virus: update interim WHO guidance on global surveillance. Available from: http://www.who.int/csr/disease/swineflu/WHO casedefinition swine flu 200904 29.pdf

7. CDC intensive care patient with severe novel influenza $A(H 1 N 1)$ virus infection- Michigan, June 2009, MMWR, Morb Mortal Wkly Rep 2009, 58(33):913-918

8. Kelly h, Grant K. Interim analysis of pandemic influenza (H1N1) 2009 in Australia: surveillance trends, age of infection and effectiveness of seasonal vaccination. Euro Surveill, 2009; 14(31):pii=19288. Available from: http://www.eurosurveillance.org/viewArticle.aspx?Articled=19288

9. Oleveira WK, et al, Pandemic in Brazil: Analysis of the first 34,506 cases of influenza-like illness with severe acute respiratory infection (SARI). Euro Surveill, 2009; 14(42):pii=19362. Available from: http://www.eurosurveillance.org/viewArticle.aspx?Articled $=19362$

10. Cullen G, Martin J, Boland M; et al. Surveillance of the First 205 confirmed hospitalized cases of pandemic H1N1 influenza in Ireland, 28 April-3October 2009. Euro Surveill, 2009; 14(44):pii=19389. Available from: http://www.eurosurveillance.org/viewArticle.aspx?Articled=19389

11. van 't Klooster TM, Wielders CC, Donker T, Isken $L$, Meijer A, van den Wijngaard CC, van der Sande MA, van der Hoek W. Surveillance of Hospitalisations for 2009 Pandemic Influenza $A(H 1 N 1)$ in the Netherlands, 5 June - 31 December 2009. Euro Surveill. 2010;15(2):pii=19461. Available from: http://www.eurosurveillance.org/ViewArticle.aspx?Articleld=19461

12. Sabbe M, Hue D, Antoine J, Dupont $Y$, Van Eldere J, Van Ranst M, Thomas I. Influenza surveillance in children: first experiences with the Belgian Paediatric Surveillance system "PediSurv". Arch Public Health 2010; 68(3): 94-9

13. Louie JK, Acosta M, Winter K, et al. Factors associated with death or hospitalization Due to Pandemic 2009 Influenza A (H1N1) Infection in California. JAMA, 2009; 302(17): 1896-1902.

14. Fuhrman C, Bonmarin I, Paty AC et al. Severe hospitalized 2009 pandemic influenza A (H1N1) cases in France, 1 July-15 November 2009. Euro Surveill, 2010; 15(2):pii=19463. Available from: http://www.eurosurveillance.org/viewArticle.aspx?Articled=19463

15. Baker MG, Kelly H, Wilson N. Pandemic H1N1 influenza lessons from southern hemisphere. Euro Surveill, 2009; 14(42):pii=19370. Available from: http://www.eurosurveillance.org/viewArticle.aspx?Articled $=19370$

16. Belgian National Influenza Centre. Weekly epidemiological report influenza. Scientific Institute of Public Health; 2009. Available from: http://www.iph.fgov.be/flu-surveillance 